\title{
Analysis of ICP Variants for the Registration of Partially Overlapping Time-of-Flight Range Images
}

\author{
Robert L. Larkins, Michael J. Cree and Adrian A. Dorrington \\ School of Engineering, University of Waikato, New Zealand \\ Email: rll6@students.waikato.ac.nz
}

\begin{abstract}
The iterative closest point (ICP) algorithm is one of the most commonly used methods for registering partially overlapping range images. Nevertheless, this algorithm was not originally designed for this task, and many variants have been proposed in an effort to improve its proficiency. The relatively new full-field amplitude-modulated time-of-flight range imaging cameras present further complications to registration in the form of measurement errors due to mixed and scattered light. This paper investigates the effectiveness of the most common ICP variants applied to range image data acquired from full-field range imaging cameras. The original ICP algorithm combined with boundary rejection performed the same as or better than the majority of variants tested. In fact, many of these variants proved to decrease the registration alignment.
\end{abstract}

Keywords: ICP, AMCW, registration, range image, point cloud

\section{Introduction}

Range imaging systems are commonly used to acquire range data of $3 \mathrm{D}$ scenes. There are a variety of systems capable of range data acquisition [1], including structured lighting, stereoscopic triangulation and active illumination time-of-flight (TOF) systems. A relatively new contender are the range imaging cameras which perform simultaneous fullfield range acquisitions by amplitude modulated continuous wave (AMCW) time-of-flight methods $[2,3]$, herein referred to as range imaging cameras. The advantage of range imaging cameras is that they acquire range data simultaneously across the field-of-view (FOV), thus are very efficient and simple to use. Their disadvantage (other than low resolution) is that they are susceptible to measurement errors due to scattered or mixed light from multiple sources at different ranges. These can lead to substantially inaccurate range values, particularly at pixels on the boundaries of objects.

Range data acquisition systems, including range imaging cameras, collect range data to the scene from a single view point, and must be moved to various vantage points to acquire a dataset that fully encompasses the scene. Registration is required to align multiple views of the scene together, putting all the range data into one coordinate system. The iterative closest point (ICP) algorithm [4, $5]$ and its variants are most often used to perform

978-1-4244-9631-0/10/\$26.00 @2010 IEEE this registration. Because of the limitations in the ICP algorithm many variants on the base ICP algorithm have been proposed.

This paper is concerned with the ICP algorithm and its variants for registering multiple views of range data captured with a range imaging camera. The ICP algorithm and its variants has been evaluated previously $[6,7]$ (and is discussed further below) but these evaluations have not used range data from a range imaging camera. That is, it is not known how robust the ICP variants are to the errors that occur in such range data. Because these errors are fundamentally different in nature to those found in other range imagers, the registration may be affected in markedly different ways.

The errors in range data captured with range imaging cameras occur due to the nature of the capturing process and include mixed pixels [8], multipath effects, specular reflection and ambiguous points [9]. Each pixel in the sensor has a fixed FOV from which it collects light. When the FOV encompasses multiple reflecting surfaces a pixel becomes mixed as each surface reflects back light and mixes multiple phases to produce an anomalous point. In contrast, multipath effects are caused by global light transport, namely when scattered light that originates from outside the pixel's FOV is received by the pixel and corrupts the measured range. Specular reflections, when they occur, often cause a pixel to saturate and ruin range measurement. Finally, the ambiguity problem occurs when 
a measured object is further away than the ambiguity distance; the distance at which the phase of the received modulated signal repeats. The measured range is therefore modulo the ambiguity distance of the actual range.

The ICP algorithm introduced by Chen and Medioni [4], and Besl and McKay [5], was designed to register a captured point cloud with a predefined model, and has since been expanded to register partially overlapping range images. Many variants have been proposed for the ICP algorithm to improve its registration capability. The literature by Rusinkiewicz and Levoy [6] and Salvi et al. [7] investigate these variants using simulated data. This paper expands on their work by testing whether the most common variants do in fact improve the capability of the ICP algorithm when registering range images contaminated to some degree by the deleterious effects unique to full-field AMCW range image acquisition.

In Section 2 we review previous work carried out to analyse ICP variants and perform range image registration to better isolate the limitations of those studies. In Section 3 the tested ICP algorithm and variants are summarised. That is followed by Section 4 which outlines the tests performed and the methodology used to analyse the efficacy of the ICP variants for registering range imaging camera data, and is topped off with the results and discussion in Sections 5 and 6, respectively.

\section{Background}

The ICP algorithm and its subsequent variants have become the predominant class of algorithms for registering range images. Rusinkiewicz and Levoy [6] and Salvi et al. [7] explored many of these variants to determine their convergence speed and their alignment accuracy, with testing performed using fully overlapping simulated data. Their findings indicate that Chen's [4] approach is more robust than Besl's [5], but is less appropriate in the presence of non-overlapping regions.

Although range imaging cameras are gaining popularity, there has been minimal work focused on registering the images they capture and in particular their use for modelling a 3D scene. Fuchs and May [10] perform surface reconstruction using a SwissRanger 3000 camera attached to a robot arm. The arm's position provided the initial camera pose, which was further refined using ICP. May et al. [11] carried out a deeper analysis of the SwissRanger 3000 for 3D mapping, in particular, investigating its robustness for use with autonomous mobile robots. Swadzba et al. [12] proposed a fully automatic system for constructing a scene using AMCW range images. Their system uses a variety of algorithms to filter and register each image, thereby reducing the registration error, but at the expense of increased computation. Feulner et al. [13] presented an algorithm combining both the captured range and intensity data for modelling static scenes; in their work they test the capability of camera motion prediction, projection ICP, multiresolution hierarchy and random search, finding that each approach makes the registration more robust, although projection ICP only improved certain scenes.

\section{ICP Algorithm and Variants}

The ICP algorithm consists of six distinct stages, each composed of algorithmic variants that perform a specific task. These stages are iterated over until convergence of the registration. Assuming two point clouds to be registered the stages are:

1. Selection of a set of points from one or both point clouds.

2. Matching each selected point to a corresponding point in the other cloud.

3. Weighting each point-pair correspondence.

4. Rejection of certain correspondences based on some criteria.

5. Error Metric based on corresponding points.

6. Minimisation of the error metric.

Throughout this paper, the terminology of fixed image and loose image is used to describe the two point clouds, where the fixed image is fixed in space and the loose image is freely movable by rotation and translation. The goal is to find a rotation and translation that registers the loose image with the fixed image.

The ICP variants are now addressed according to the matching, weighting and rejection stages.

\subsection{Matching}

The matching stage identifies which points on the fixed image correspond with the points selected from the loose image. Three variants are tested for matching ability.

The closest point algorithm [5] is one of the most common approaches. The Euclidean distance from a selected point in the loose image and every point in the fixed image is calculated, with the closest point in the fixed image chosen to match.

An alternative approach that builds on the closest point matching first takes the closest point found on the fixed image and fits a plane tangent to the local surface at the fixed image point. The new correspondence is the point on this plane that is 
closest to the initially selected loose image point. The goal is to more accurately determine where the point from the loose image occurs on the fixed image. This is referred to as plane intersect henceforth.

The method proposed by Chen and Medioni [4] and utilised by Dorai et al. [14] uses the plane intersect, but differs in that the new correspondence is the intercept point of this plane with the ray normal to the surface at the selected loose image point. This is referred to as normal intersect henceforth.

\subsection{Weighting}

The impact that each point-pair correspondence has on the registration can be modified by giving each correspondence a weighting. We explore three common weighting methods. The first is uniform weighting as used in the baseline algorithm.

The second method is to allocate correspondences a lower weight $w$ the greater the Euclidean distance between points. This is achieved by

$$
w=1-\frac{\left|\mathbf{p}_{1}-\mathbf{p}_{2}\right|}{m}
$$

where $\mathbf{p}_{1}$ and $\mathbf{p}_{2}$ are the two corresponding points and $m$ is the greatest distance found between all corresponding points. This weighting is designed to reduce the influence that point-pairs have at greater distances from the centre of the image, while still allowing them to contribute to the registration.

The third method calculates the similarity between the normals to the local surfaces at the two corresponding points, namely the weight $w$ is given by

$$
w=\mathbf{n}_{1} \cdot \mathbf{n}_{2}
$$

where $\mathbf{n}_{1}$ and $\mathbf{n}_{2}$ are the two normals.

\subsection{Rejection}

The use of rejection is a more definitive approach to dealing with point correspondences, and is usually used for removing outliers. This strategy of rejecting certain correspondences completely removes any effect they have on the minimisation stage. We test five rejection algorithms to determine if they enhance the registration.

A common strategy for removing outliers is to reject correspondences with the greatest Euclidean distance. Rejecting the worst $10 \%$ is a common approach $[6,15]$. An alternative is to reject outliers whose correspondence distances are greater than 2.5 times the standard deviation $[6,14]$.

The registration of partially overlapping range images introduces the problem of creating correspondences that are paired with points in non-overlapping regions; the use of boundary rejection (Section 4.2) overcomes this issue. Another method of dealing with partial overlap is to reject all correspondences for each point in the fixed image except the one with the smallest distance [12]. We refer to this algorithm as unique, and it is used in combination with boundary rejection.

Rejection based on the angular difference in the corresponding normals has also been used [6]. Typically if the angle between the two normals is greater than $45^{\circ}$ then the correspondence is rejected.

The fifth approach rejects correspondences if either of the points are mixed. This is specifically targeting erroneous pixels that only occur in data captured with full-field range imaging cameras. A point is identified as mixed if the normal of the surface at this point is close to being perpendicular to the ray from the camera [16].

\section{Materials and Methods}

All range images for testing were captured with a SwissRanger 4000 [17] at a resolution of $144 \times 176$ pixels. Each capture was the average of 200 frames.

\subsection{Test Scenes}

We tested the ICP algorithm on two real scenes. The first scene is a stair shaped structure placed sideways on the ground. This scene has many flat surfaces making for a very structured geometry. Because each step has a uniform shape an additional level of difficulty is introduced. Two sets of captures were taken of the stairs, one including views that were designed to minimise the presence of mixed pixels, Figure 1(a), and the other, Figure $1(\mathrm{~b})$, with views that maximise the number of mixed pixels. This is to provide a comparison between scenes consisting of extensive mixed pixels and scenes without mixed pixels.

The second scene, Figure 1(c), is of a frog ornament sitting on an undulating sheet. This scene was designed to remove any edges and to minimise the number of flat surfaces. It contains a variety of surface shapes including obscured portions of the scene that introduce mixed pixels.

Each scene is captured from a variety of view points, one for which the scene is solely rotated and another in which the scene is solely translated. The rotation is tested at five angles, $2^{\circ}, 5^{\circ}, 10^{\circ}, 20^{\circ}$ and $50^{\circ}$ from the initial capture. The overlap between each subsequent rotation was maximised by aiming the camera at the same location in an effort to minimise any translation, though each subsequent rotation marginally decreases the overlap due to the cameras FOV. The scene was also captured 


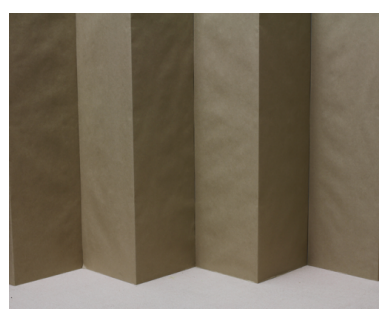

(a) Stairs

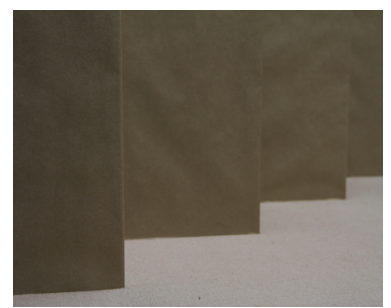

(b) Mixed Pixel Stairs

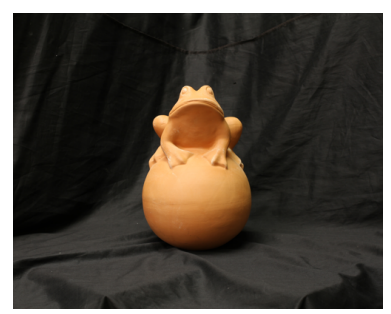

(c) Frog Ornament

Figure 1: The scenes used to test each variant. Figure 1(a) is designed to minimise the occurrence of mixed pixels, while Figure 1(b) maximises their occurrence.

by translating the camera without rotation so that two adjacent images overlap by approximately $95 \%$, $90 \%, 75 \%$ and $50 \%$.

\subsection{Registration Baseline}

The scope of this undertaking would be enormous if every possible combination of variants was tested, therefore a baseline algorithm is used that only contains the core requirements necessary for ICP registration. The capability of each variant is then investigated in relation to the following baseline:

- Every point on the loose image is used for registration.

- A loose image point corresponds with the closest point on the fixed image.

- Every point-pair correspondence has the same weight.

- Boundary rejection of point-pairs.

- Quaternion based minimisation.

The only algorithm variant included as part of the baseline and applied to all testing is boundary rejection, which rejects any point-pairs whose correspondences go within two points of the fixed image's edge. Boundary rejection is an important step for registering partially overlapping range images [6, 15, 18], because if non-overlapping points contribute to the registration they tend to pull the two range images closer together, substantially decreasing their ability to align correctly.

\subsection{Testing Restrictions}

The testing of the ICP variants is restricted to 30 iterations as initial testing showed that variants plateau by this iteration. Throughout testing, the primary focus is on the matching, weighting and rejection variants. The selection stage uses all points in the loose image, giving each point the potential to contribute to the registration. Typically the number of points is limited to reduce the computational time, but due to the comparatively low resolution of the range imager, it is feasible to use all available points.

Various closed-form solutions have been used to minimise the sum of the squared distances between corresponding points. In an evaluation of these solutions, Eggert et al. [19] found that there is no significant difference between their capability, we therefore use quaternions [20] for the error metric and minimisation.

\subsection{Measure of Error}

The ability of each variant is determined by testing how well it aligns two range images. This is achieved by finding the shortest distance between a loose image point and the plane fitted to the closest corresponding point on the fixed image. The measure of error is then calculated as the root mean square error (RMS) of every point-plane distance. Because we are registering partially overlapping range images, measuring the distance between the non-overlapping regions does not portray the true error, therefore correspondences that connect to within two points of the fixed image's edge are removed. The total error $\varepsilon$ is given by

$$
\varepsilon=\sqrt{\frac{1}{n} \sum_{i=1}^{n}\left(\left(\mathbf{L}_{i}-\mathbf{F}_{i}\right) \cdot \hat{\mathbf{N}}_{i}\right)^{2}}
$$

where $n$ is the number of point-pairs, $\mathbf{L}$ and $\mathbf{F}$ are two corresponding points on the loose and fixed image, respectively, and $\hat{\mathbf{N}}$ is the unit normal at $\mathbf{F}$ of the fixed image's surface. This approach was chosen over the actual corresponding point distance as it calculates a better estimate of the distance between a point on the loose image and the surface of the fixed image.

\section{Results}

Results are presented on the registration of the rotational and the translational captures respect- 
ively. It was found that all variants were incapable of registering scenes when there was a $50^{\circ}$ rotation between images or when the overlap decreased to $75 \%$. Results are only presented for rotations and translations smaller than these respective limits. Registration run time took approximately one second per iteration, and was carried out using MATLAB R2009a on an Intel Core 2 Duo 3GHz. The results show that the number of iterations can be decreased to approximately 10 without significantly impacting the accuracy.

\subsection{Matching}

The three matching algorithms were tested first and are presented in Figure 2. The plane intersect algorithm had the best performance at $2^{\circ}$ rotation for each scene, while the baseline generally had the worst performance. The baseline was however able to deal with the mixed stairs scene more reliably than the normal intersect. For a $5^{\circ}$ rotation all three algorithms have a very similar performance for each scene. For $10^{\circ}$ and $20^{\circ}$ rotations the baseline and plane intersect continue to have the same ability, while the normal intersect becomes erratic, generally having a lower performance and in the case of the frog scene, failing at $20^{\circ}$.

All three algorithms registered the stairs scene correctly with equal performance when there was a 95\%, Figure 2(d), and 90\% overlap. The normal intersect has a spike at the seventh iteration and was likely due to incorrectly matched points. The baseline and plane intersect have the same ability for the frog scene, but the normal intersect did not work for either overlap.

\subsection{Weighting}

The effect on registration of the distance and normal weightings was minimal for a two degree shift between range images, with each having equivalent performance as shown in Figures 3(a) and 3(b). However, for the mixed stairs scene, Figure 3(c), their registration ability drops in relation to the baseline algorithm. As the angle of separation increased, the baseline algorithm performed equal to or better than both of the weightings for all scenes.

The registration performance for translations was similar to rotations, with the baseline algorithm being marginally better than the two weighting methods for both $95 \%$ and $90 \%$ overlap. The frog scene with a $95 \%$ overlap is shown in Figure 3(d).

\subsection{Rejection}

The results of testing the rejection algorithms are shown in Figure 4. For a rotation of $2^{\circ}$ the worst $10 \%$ rejection had the lowest performance, while



(a) Frog scene: $2^{\circ}$ rotation.

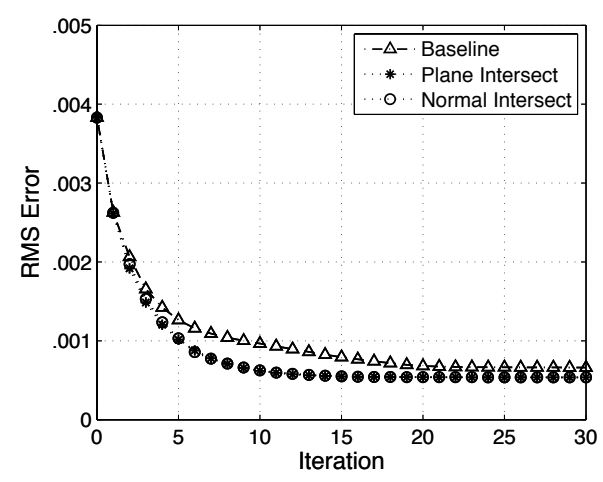

(b) Stairs scene: $2^{\circ}$ rotation.

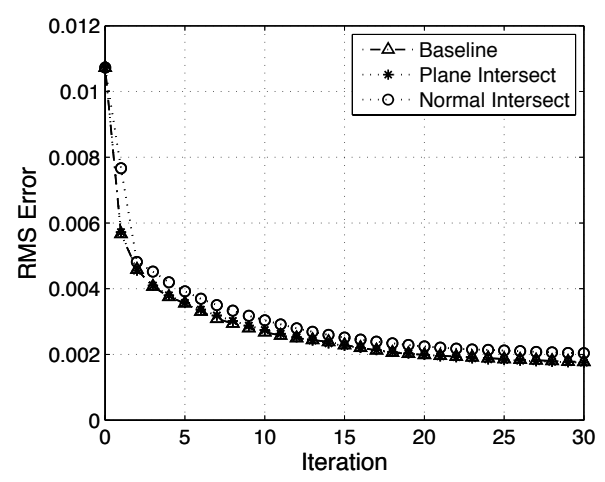

(c) Mixed stairs: $2^{\circ}$ Rotation.

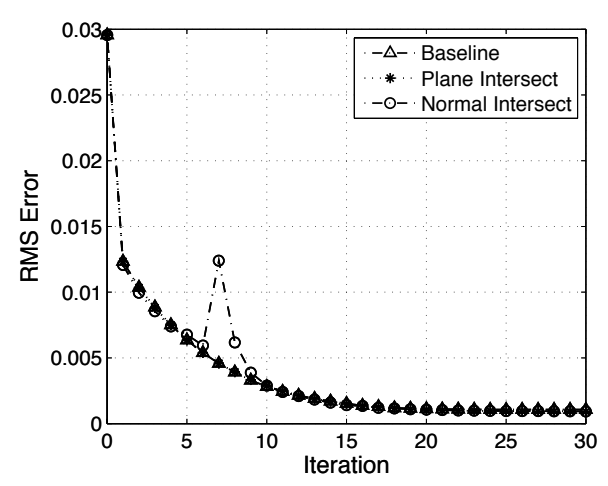

(d) Stairs scene: $95 \%$ overlap.

Figure 2: The error of the registration of the three matching algorithms when used with the smallest rotation $\left(2^{\circ}\right)$ or translation (95\% overlap). 




(a) Frog scene: $2^{\circ}$ rotation.

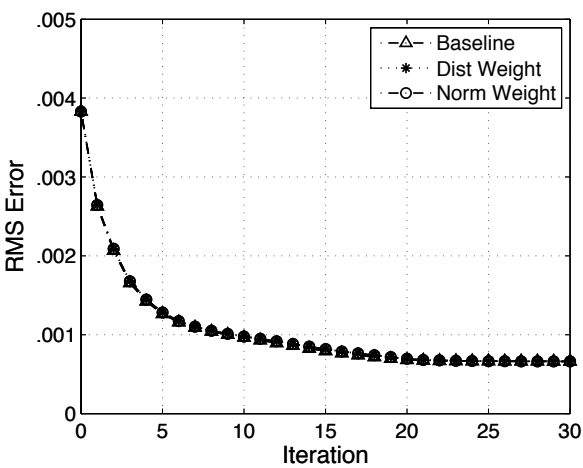

(b) Stairs scene: $2^{\circ}$ rotation.

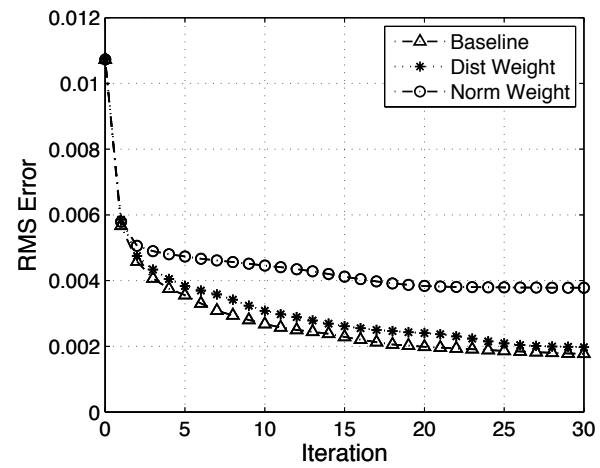

(c) Mixed stairs: $2^{\circ}$ rotation.

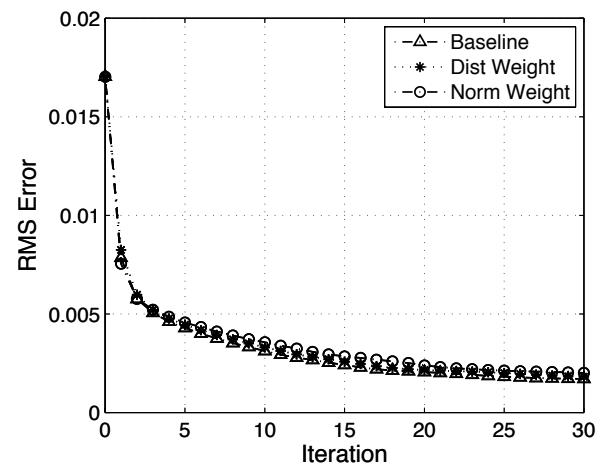

(d) Frog scene: $95 \%$ overlap.

Figure 3: The error of the registration for two weighting algorithms and the baseline algorithm when used with the smallest rotation and translation. the remaining algorithms performed equally. The baseline algorithm performs the best for the mixed stairs scene, Figure 4(c). As the angular displacement increased, the baseline continued to achieve better alignment than the other algorithms, though normal rejection performed slightly better at $20^{\circ}$ when used with the non-mixed scenes. For the mixed stairs scene the baseline consistently achieved the best result, with no pattern emerging to indicate the order of their ability.

For translation the results showed that rejecting the worst $10 \%$ had the slowest convergence, though by iteration 30 all algorithms performed equally when used with a $95 \%$ overlap. This trend continued with a $90 \%$ overlap, though normal rejection gave a marginal improvement initially.

\section{Discussion}

The most distinct finding was that the baseline algorithm performed the same as or better than the majority of tested algorithms for both the rotation and translation of each scene. There were however circumstances in which variants such as the plane intersect and normal rejection improved the registration, but there was no consistent improvement in performance. Because range imaging cameras can operate at speeds of greater than 30 FPS, in a moving camera situation the difference between each successive range image is generally small, therefore we primarily focussed on the $2^{\circ}$ rotation and $95 \%$ overlap. Given these conditions only the plane intercept likely decreases the measured error for all potential scenes. These results though are not indicative for registering range images captured using other systems, as the range images are likely to have different characteristics.

The presented results indicate that the variants have a similar ability for both full-field AMCW range images and the simulated data used by $\mathrm{Ru}-$ sinkiewicz and Levoy [6]. Salvi et al. [7] used simulated data to test only the closest-point and normal intersect algorithms. Rusinkiewicz and Levoy did not specify what rotation and translation difference was used between the point clouds and therefore the true correlation between results is unknown. Salvi et al. however use a $5^{\circ}$ offset around each axis and also tests their data with 95\%, 90\%, $80 \%$ and $50 \%$ overlap. The closest-point and normal intersect algorithms produce results that is similar for both our AMCW data and the simulated data at smaller rotation and translation offsets, though these results vary depending on the images being registered. As the overlap decreases the normal intersect algorithm continues to perform the best with the simulated data. This differs from our results which show the closest-point algorithm pro- 


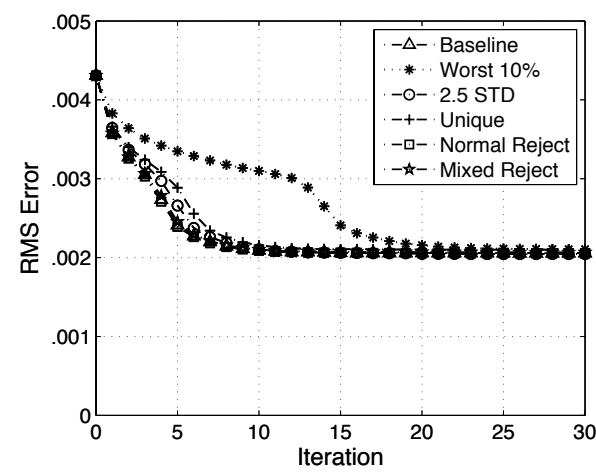

(a) Frog scene: $2^{\circ}$ rotation.

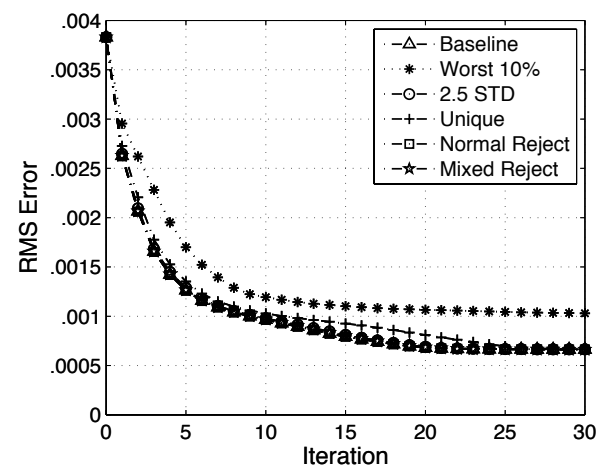

(b) Stairs scene: $2^{\circ}$ rotation.

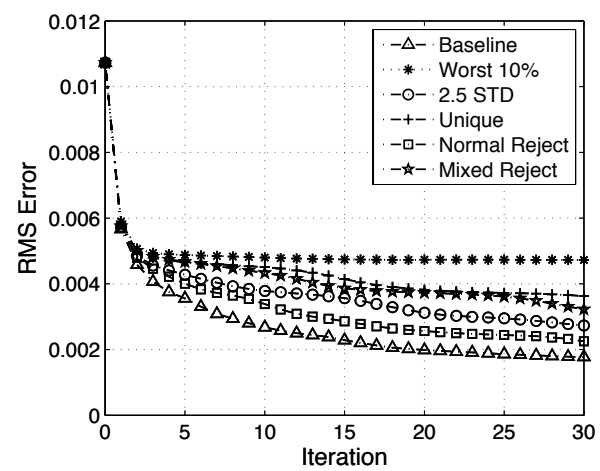

(c) Mixed stairs: $2^{\circ}$ rotation.

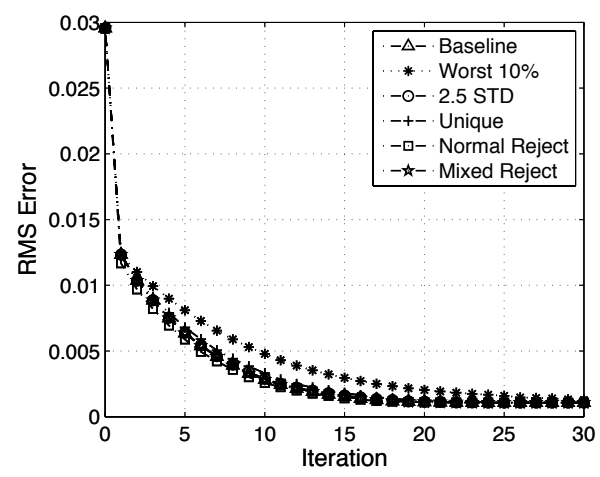

(d) Stairs scene: $95 \%$ overlap.

Figure 4: These graphs show the ability of the five rejection algorithms in comparison with the baseline when used with the smallest rotational and translational shift. ducing better alignment. The plane intersect algorithm was not tested by either Rusinkiewicz and Levoy or Salvi et al. The simulated and real data produced similar results for both the weighting and rejection variants, which like our work is shown to provide no significant advantage.

Decreasing the measured error is not the only metric, as this rate of capture is not useful if the selected algorithm is unable to register the images in real-time. This is where changes to initial selection of points and the rejection of correspondences is useful for reducing the number of points used for the minimisation. The reduction of points used has a relative decrease in the processing time required to perform the registration, but keeping the points necessary for a correct alignment is a subsequent task that is outside the scope of this paper.

It was found that the baseline was robust in the presence of mixed pixels at a smaller rotation and translation, with their removal improving registration for the frog scene at greater levels of translation. The opposite effect was observed when sections of the stairs were obscured, presumably because mixed pixels helped fill in these gaps, giving the ICP algorithm points to register these empty areas against. However, there is a significant difference in the RMS error between the two stair scenes, with the clean stairs aligning with much greater accuracy, showing that a scene without mixed pixels will tend to have better registration alignment.

In relation to the translation, it becomes increasingly more difficult for the ICP algorithm to register partially overlapping range images that are initially layered on top of each other. This is because the ICP algorithm is not designed for use with non-overlapping datasets. The use of matching criteria designed for this task would likely help improve the registration of images that have a translational difference.

\section{Conclusion}

The registration of range images is not a trivial task, with many variations to the ICP algorithm proposed in the literature since its inception. This paper investigated a selection of the more common variants to determine whether they improve or detract the capability of the ICP algorithm when used to register range images captured with a fullfield AMCW TOF ranging system.

The analysis of these variants found that they rarely provided any advantage over the baseline ICP algorithm, with some variants actively diminishing the registration ability. The produced results also showed that the capability of ICP tended to be limited to a rotation of $20^{\circ}$ and an overlap of $90 \%$. 


\section{Acknowledgements}

Robert Larkins acknowledges the financial support provided by both the Range Imaging and Waikato Doctoral Scholarships.

\section{References}

[1] F. Blais, "Review of 20 years of range sensor development," Journal of Electronic Imaging, vol. 13, no. 1, pp. 231-243, January 2004.

[2] MESA Imaging, "SR4000 Data Sheet Revision 4.6," June 2010, http: //www.mesa-imaging.ch/dlm.php?fname= pdf/SR4000_Data_Sheet.pdf, accessed: 23rd June 2010.

[3] PMD Technologies, "PMD[vision] (B)CamCube 3.0," June 2010, http://www. pmdtec.com/fileadmin/pmdtec/downloads/ documentation/datenblatt_camcube3.pdf, accessed: 23rd June 2010.

[4] Y. Chen and G. Medioni, "Object modeling by registration of multiple range images," in IEEE International Conference on Robotics and Automation, vol. 3, 1991, pp. 2724-2729.

[5] P. J. Besl and N. D. McKay, "A method for registration of 3-D shapes," IEEE Transactions on Pattern Analysis and Machine Intelligence, vol. 14, no. 2, pp. 239-256, 1992.

[6] S. Rusinkiewicz and M. Levoy, "Efficient variants of the ICP algorithm," in Third International Conference on 3-D Digital Imaging and Modeling (3DIM'01), 2001, pp. 145-152.

[7] J. Salvi, C. Matabosch, D. Fofi, and J. Forest, "A review of recent range image registration methods with accuracy evaluation," Image and Vision Computing, vol. 25, no. 5, pp. 578596, 2007.

[8] M. Hebert and E. Krotkov, "3D measurements from imaging laser radars: how good are they?" Image and Vision Computing, vol. 10, no. 3, pp. 170-178, April 1992.

[9] A. A. Dorrington, M. J. Cree, A. D. Payne, R. M. Conroy, and D. A. Carnegie, "Achieving sub-millimetre precision with a solid-state full-field heterodyning range imaging camera," Measurement Science and Technology, vol. 18, no. 9, pp. 2809-2816, 2007.

[10] S. Fuchs and S. May, "Calibration and registration for precise surface reconstruction with time-of-flight cameras," International Journal of Intelligent Systems Technologies and Applications, vol. 5, no. 3-4, pp. 274-284, 2008.
[11] S. May, D. Droeschel, D. Holz, S. Fuchs, E. Malis, A. Nüchter, and J. Hertzberg, "Three-dimensional mapping with time-offlight cameras," Journal of Field Robotics, vol. 26, no. 11-12, pp. 934-965, 2009.

[12] A. Swadzba, B. Liu, J. Penne, O. Jesorsky, and R. Kompe, "A comprehensive system for 3D modeling from range images acquired from a 3D ToF sensor," in The 5th International Conference on Computer Vision Systems, 2007.

[13] J. Feulner, J. Penne, E. Kollorz, and J. Hornegger, "Robust real-time 3D modeling of static scenes using solely a timeof-flight sensor," in IEEE Computer Society Conference on Computer Vision and Pattern Recognition Workshops, 2009, pp. 74-81.

[14] C. Dorai, G. Wang, A. K. Jain, and C. Mercer, "Registration and integration of multiple object views for $3 \mathrm{~d}$ model construction," IEEE Transactions on Pattern Analysis and $M a-$ chine Intelligence, vol. 20, no. 1, pp. 83-89, 1998.

[15] K. Pulli, "Multiview registration for large data sets," in Second International Conference on 3-D Digital Imaging and Modeling. IEEE Computer Society, 1999, pp. 160-168.

[16] R. L. Larkins, M. J. Cree, A. A. Dorrington, and J. P. Godbaz, "Surface projection for mixed pixel restoration," in Proceedings of the 24th International Image and Vision Computing New Zealand Conference (IVCNZ 2009), 2009, pp. 431-436.

[17] B. Büttgen, T. Oggier, M. Lehmann, R. Kaufmann, and F. Lustenberger, "CCD/CMOS lock-in pixel for range imaging: challenges, limitations and state-of-the-art," Proceedings of the 1st Range Imaging Research Day, pp. 21-32, June 2005.

[18] G. Turk and M. Levoy, "Zippered polygon meshes from range images," in Proceedings of the 21st International SIGGRAPH Conference. ACM, 1994, pp. 311-318.

[19] D. Eggert, A. Lorusso, and R. Fisher, "Estimating 3-D rigid body transformations: a comparison of four major algorithms," Machine Vision and Applications, vol. 9, no. 5-6, pp. 272-290, March 1997.

[20] B. K. P. Horn, "Closed-form solution of absolute orientation using unit quaternions," Journal of the Optical Society of America A, vol. 4, no. 4, pp. 629-642, 1987. 